\section{Integrative oncology - strong science is needed for better patient care}

\author{
Heather Greenlee, Suzanna M. Zick, David Rosenthal, Lorenzo Cohen, \\ Barrie Cassileth and Debu Tripathy
}

\begin{abstract}
We read David Gorski's Opinion article on integrative oncology (Integrative oncology: really the best of both worlds? Nature Rev. Cancer 14, 692-700 (2014) $)^{1}$ with both interest and dismay. We firmly agree with Gorski that all forms of medical practice should be based on evidence supporting safety and efficacy. In addition, we concur that the field of integrative oncology encompasses a wide range of therapeutic approaches that vary in their level of evidence. However, we are concerned that Gorski's article selectively focuses on practices with the weakest mechanistic
\end{abstract} evidence base (for example, homeopathy and reiki) and, by doing so, ignores the current body of evidence that demonstrates where integrative oncology can be the most useful.

In oncology, integrative therapies are often used and tested in conditions that are not well-controlled by conventional approaches (for example, fatigue, pain and anxiety). Poor control of cancer symptoms and side effects of treatment are associated with non-adherence or discontinuation of standard oncology treatments ${ }^{2-4}$, which can decrease cancer survival ${ }^{5}$. Patients and clinicians need clear guidance on which integrative approaches can be useful to manage and control cancer-related symptoms.

The Society for Integrative Oncology (SIO) recently published comprehensive clinical guidelines on the use of integrative therapies for symptom management among patients with breast cancer in an issue of Journal of the National Cancer Institute Monograph on integrative oncology ${ }^{6}$. The clinical guidelines are based on a systematic review of randomized controlled clinical trials using rigorous methods put forth by the Institute of Medicine $\mathrm{e}^{7}$ and a grading system developed by the US Preventive Services Task Force $^{8}$. More than 80 separate therapies were assessed, and the guidelines are organized by symptoms to help guide clinical decision making. There is high-level Grade A evidence recommending routine use of meditation, yoga and relaxation with imagery to control depression and mood disorders. The same practices are also recommended for routine use with Grade B evidence to reduce stress, depression and fatigue. Acupuncture received a Grade B and can be recommended to most patients to control chemotherapy-induced nausea and vomiting. More than 30 interventions received a Grade $\mathrm{C}$ recommendation that suggested weaker evidence of benefit due to either conflicting study results or small sample sizes. Seven therapies are not recommended due to lack of effect, and one was found to be harmful. All others lacked sufficient evidence to form a recommendation. These guidelines will provide patients, clinicians and policy makers with the evidence needed to make informed treatment decisions in the care of patients with breast cancer.

Importantly, Gorski's article fails to acknowledge the public health significance of integrative oncology. In the United States, $65-75 \%$ of cancer survivors use at least one form of a complementary or an integrative therapy, often without the supervision of a physician ${ }^{9,10}$. The associated cost is not insubstantial and in 2012 was estimated at US\$9 billion per year in out-of-pocket costs ${ }^{10}$. Clearly, science is not always the driver of how individuals make health-related decisions. It is incumbent on medical institutions to discuss all options with patients so that they can make informed and wise choices. Integrative oncology programmes provide patients with access to trained integrative medicine clinicians, who collaborate closely with conventional clinicians and become part of the medical team. These programmes also provide a venue to discuss healthy lifestyle modifications (for example, diet, physical activity and weight management) that may improve cancer outcomes, while improving other health parameters related to cardiovascular and metabolic disorders. Integrative oncology programmes also serve an important role in conducting the necessary clinical research to further the knowledge base for better oncology care. The absence of integrative oncology programmes forces patients to coordinate their own care using interventions that may be dangerous, limits the infrastructure to conduct much needed clinical trials, and leaves patients and clinicians to make uninformed clinical decisions.

Modern comprehensive cancer care extends beyond merely changing the biology of cancer cells. In addition to improving clinical outcomes, the purpose of integrative oncology programmes is to ease the suffering of patients with cancer, which is as worthy a goal as shrinking the tumour. The SIO breast cancer clinical guidelines ${ }^{6}$ provide evidence that there is a large enough body of literature to systematically evaluate what works, what does not work, and where more research is needed; the evidence can then help guide clinical decisions. In the cases where there is an absence of clear clinical research data providing guidance, patients and clinicians need to engage in shared decision-making when assessing the risk-benefit ratio for each therapy.

Heather Greenlee is at the Department of Epidemiology, Mailman School of Public Health, Columbia University, New York, New York 10032, USA.

Suzanna M. Zick is at the Department of Family Medicine, University of Michigan, Ann Arbor, Michigan 48109, USA.

David Rosenthal is at Harvard Medical School and the Dana-Farber Cancer Institute, Boston, Massachusetts 02115, USA.

Lorenzo Cohen is at the Departments of General Oncology and Behavioural Science, University of Texas MD Anderson Cancer Center, Houston, Texas 77030, USA

Barrie Cassileth is at the Memorial Sloan Kettering

Cancer Center, New York, New York 10021, USA.

Debu Tripathy is at the Department of Breast Medical Oncology, University of Texas MD Anderson Cancer Center, Houston, Texas 77030, USA.

H.G. is the Immediate Past President of the Society for Integrative Oncology, S.Z. currently serves as the President, and all other authors are Past Presidents of the Society. Correspondence to H.G. e-mail: hg2120@columbia.edu doi: $10.1038 /$ nrc3822-c1

1. Gorski, D. Integrative oncology: really the best of both worlds? Nature Rev. Cancer 14, 692-700 (2014).

2. Kidwell, K. M. et al. Patient-reported symptoms and discontinuation of adjuvant aromatase inhibitor therapy. Cancer 120, 2403-2411 (2014).

3. Walker, M. S. et al. Early treatment discontinuation and switching in first-line metastatic breast cancer: the role of patient-reported symptom burden. Breast Cancer Res. Treat. 144, 673-681 (2014).

4. Chim, K. et al. Joint pain severity predicts premature discontinuation of aromatase inhibitors in breast cancer survivors. BMC Cancer 13, 401 (2013).

5. Hershman, D. L. et al. Early discontinuation and nonadherence to adjuvant hormonal therapy are associated with increased mortality in women with breast cancer. Breast Cancer Res. Treat. 126, 529-537 (2011).

6. Greenlee, H. et al. Clinical practice guidelines on the use of integrative therapies as supportive care in patients treated for breast cancer. J. Natl Cancer Inst. Monogr. 50, 346-358 (2014).

7. Institute of Medicine. Clinical Practice Guidelines We Can Trust (The National Academies Press, 2011).

8. Grade definitions. U.S. Preventive Services Task Force [online], http://www.uspreventiveservicestaskforce.org/ uspstf/grades.htm (2014).

9. Mao, J. J. et al. Complementary and alternative medicine use among cancer survivors: a populationbased study. J. Cancer Surviv. 5, 8-17 (2011).

10. John, G. et al. Costs associated with complementary and alternative medicine use among cancer survivors in the United States: results from the 2012 National Health Interview Survey. J. Altern. Complement. Med. 20, A4 (2014).

Competing interests statement

The authors declare no competing interests. 